\title{
Aplicação de métricas da Análise de Redes Sociais como apoio a avaliação das interações discentes em fóruns de discussão online de um Ambiente Virtual de Aprendizagem
}

\author{
Jarbele C. da Silva ${ }^{1}$, Alisson V. de Brito ${ }^{2}$, Francisco P. A. de Medeiros ${ }^{3}$ \\ ${ }^{1}$ Centro de Ciências Aplicadas e Educação - Universidade Federal da Paraíba (UFPB) - \\ Rio Tinto, PB - Brasil \\ ${ }^{2}$ Centro de Informática - Universidade Federal da Paraíba (UFPB) - João Pessoa, PB - \\ Brasil \\ ${ }^{3}$ Instituto Federal de Educação, Ciência e Tecnologia da Paraíba (IFPB) - João Pessoa, \\ PB - Brasil \\ jarbele.cassia@dce.ufpb.br, alisson@ci.ufpb.br, petronio@ifpb.edu.br
}

\begin{abstract}
This paper this work investigates the potential generated by the use of Social Network Analysis techniques (ARS) applied to the analysis of the interactions between students of a distance learning course that establish communication in online discussion forums, to ascertain patterns of interaction that may have influence on student learning. The results show that by applying the degree of centrality metrics, centrality intermediation and closeness centrality related to student achievement express a linear correlation between such variables, showing a trend in the performance of the same throughout the course.

Resumo. Este artigo investiga o potencial gerado pelo uso de técnicas de Análise de Redes Sociais (ARS) aplicado à análise das interações entre alunos de um curso à distância que estabelecem comunicação em fóruns de discussão online, a fim de conhecer padrões de interação que podem ter influência na aprendizagem do aluno. Os resultados obtidos evidenciam que através da aplicação das métricas de centralidade de grau, centralidade de intermediação e centralidade de proximidade relacionadas com o desempenho do aluno é obtida uma correlação linear entre tais variáveis, demonstrando uma tendência no desempenho do aluno ao longo do curso.
\end{abstract}

\section{Introdução}

O desenvolvimento de estudos referentes a cursos de educação à distância, ou simplesmente E-Learning, têm se tornado frequentes no cenário cientifico brasileiro, tendo em vista a ampla difusão dos métodos de ensino-aprendizagem no país. Várias iniciativas públicas tem sido desenvolvidas com o objetivo de incentivar a disseminação do modelo de ensino não-presencial e, atualmente, há um número crescente de cursos ministrados em ambientes de E-Learning.

Neste formato de ensino, à distância, as pessoas não estão apenas reunidas em torno de um tema. Há uma proposta a cumprir, situações didáticas e alguém do grupo 
que provoca e estimula a participação dos demais. Essas características distinguiriam as chamadas redes e comunidades virtuais de aprendizagem dos demais agrupamentos na Internet [Carvalho 2009].

Surge, então, a necessidade de se realizar uma análise mais específica dos relacionamentos, atividades e informações geradas por meio do monitoramento das interações destes agrupamentos. A Análise de Redes Sociais (Analysis Social Networks, SNA ou ARS) estuda como os comportamentos ou as opiniões dos indivíduos dependem das estruturas nas quais eles se inserem, onde a unidade de análise não são os atributos individuais (classe, sexo, idade, gênero), mas o conjunto de relações que os indivíduos estabelecem através das suas interações uns com os outros [Marteleto 2001].

É nessa contextura, perante a necessidade de se avaliar o impacto da interação de cada estudante na sua aprendizagem, que este trabalho surge para investigar e discutir o uso de métricas de Análise de Redes Sociais no acompanhamento do desempenho de alunos de um curso à distância através da interação estabelecida em fóruns de discussão online, de modo a perceber as contribuições que este tipo de análise pode fornecer ao professor, como informação para pôr em prática estratégias para um acompanhamento efetivo da aprendizagem do aluno e/ou para monitoramento e gerenciamento de cursos a distância.

O artigo está organizado na seguinte forma: na Seção 2 citamos os trabalhos relacionados ao assunto desta pesquisa. A Seção 3 apresenta a fundamentação teórica sobre análise das redes sociais e E-Learning. A Seção 4 introduz a correlação linear, seguida da Seção 5, que apresenta a descrição do experimento. Na Seção 6 apresentamos os resultados alcançados. O artigo é finalizado na Seção 7, com as conclusões e propostas para trabalhos futuros.

\section{Trabalhos relacionados}

$\mathrm{Na}$ literatura recente há poucos trabalhos que evidenciam estudos relacionados a Análise de Redes Sociais e a E-Learning [Silva et. al. 2015].

Silva (2012) apresenta as principais contribuições de um sistema, denominado “Dracula Graph Library” para a análise e caracterização das interações que ocorrem entre participantes de comunidades online, mas não realiza a análise das interações formadas. Engel (2013) discute e apresenta a aplicação de índices de ARS no âmbito de Redes de Aprendizagem Assíncronas (Asynchronous Learning Networks), e aplica as métricas de Densidade (Density), Centralidade (Centrality) e Grau (Degree) nos grafos gerados. Rabbany (2012) tem o propósito de explorar as técnicas da ARS e da mineração de dados em comunidades online com o auxílio da ferramenta Meerkat-ED, entretanto não utiliza nenhuma técnica de ARS para analisar as interações entre os participantes da rede. Gottardo (2012) demonstra as possibilidades de utilização de técnicas de análise de redes sociais (Closeness Centrality, Betweenness Centrality e Clustering), a fim de obter informações a partir da interação em fóruns de discussão. $\mathrm{O}$ estudo é realizado na plataforma Moodle e é utilizado a ferramenta Gephi para geração das redes.

Contudo, percebe-se que tais temas de estudo têm sido pouco explorados quando associados e, quando explorados, o foco abordado refere-se, em poucos casos à utilização das técnicas de ARS como instrumento de avaliação das interações existentes em ambientes que promovem o ensino à distância, ênfase dessa pesquisa. 


\section{Análise de Redes Sociais e E-Learning}

O modelo de ensino não presencial suportado por tecnologia tem se tornado uma prática cada vez mais utilizada em educação, principalmente no que se refere ao modelo de Educação a Distância. Entretanto, as ferramentas disponíveis no Ambiente Virtual de Aprendizagem (AVA), por si só, não disponibilizam estratégias para realização de uma análise precisa do acompanhamento dos processos (níveis de aprendizagem, participação, evasão etc.) que envolvem os aprendizes neste ambiente. Este só pode ser efetivado através do uso de métricas específicas de análise e avaliação.

Através da Análise de Redes Sociais é possível compreender e acompanhar de forma mais eficaz a disseminação de informações e a interação entre as pessoas que compõem a rede [Oliveira 2012], por meio do uso de métricas de ARS que possibilitam a identificação de relacionamentos, bem como, sua interpretação.

Em Análise de Redes Sociais, a rede de interação é representada por um grafo. Os membros da rede são representados pelos nós e as arestas indicam a relação existente entre estes nós. A aplicação deste conceito para o fórum de discussão online é viável, visto que é possível representar cada participante do fórum como um nó do grafo, e a relação entre eles, como arestas, interligando-os. Este tipo de ligação irá revelar os tipos de interdependência e quão fortemente um participante do fórum se refere a um outro [Silva e Figueira 2012]. As informações obtidas em cada fórum são representadas através de um grafo dirigido, onde as interações ocorrem quando um participante do fórum responde a postagem de outro participante.

\subsection{Métricas de Análise de Redes Sociais}

Com a realização de um mapeamento sistemático [Silva et. al. 2015] foram identificadas as principais métricas de ARS utilizadas para análise de interação em redes sociais online. Dentre as métricas encontradas optou-se pela aplicação, nesta pesquisa, das métricas de: Centralidade de Grau (Centrality Degree), Centralidade de Intermediação (Betweenness Centrality) e Centralidade de Proximidade (Closeneess Centrality), visto que ambas oferecem possibilidades de identificação dos atores ativos na rede, dentre outras características.

\subsubsection{Centralidade de Grau (Centrality Degree)}

Assim sendo, um indivíduo é central, quando, por seu posicionamento, recebe informações vindas da maior parte do ambiente da rede, ou seja, o número de arestas que o ligam a outros nós é o fator determinante para a identificação da centralidade de grau (Centrality Degree) do indivíduo. Em relação a um grafo direcionado, assumisse-se o grau de entrada (In-Degree), $\mathrm{d}_{\mathrm{I}}\left(\mathrm{n}_{\mathrm{i}}\right)$, e o grau de saída (Out-degree), $\mathrm{d}_{\mathrm{o}}\left(\mathrm{n}_{\mathrm{i}}\right)$, de acordo com o direcionamento das arestas que chegam ou partem do nó. A Equação 1 define a centralidade de grau.

$$
C_{D}\left(n_{i}\right)=d\left(n_{i}\right)=\sum_{i=1}^{g} x_{i j}=\sum_{j=1}^{g} x_{j i}
$$

Equação 1. Equação para Centralidade de Grau 
V Congresso Brasileiro de Informática na Educação (CBIE 2016)

Anais dos Workshops do V Congresso Brasileiro de Informática na Educação (CBIE 2016)

\subsubsection{Centralidade de Intermediação (Betweenness Centrality)}

De acordo com Gottardo e Noronha (2012), a centralidade de intermediação (Betweenness Centrality) calcula o quanto um ator atua como "ponte", facilitando o fluxo de informação em uma determinada rede. Um ator pode estabelecer poucos elos, mas possuir uma importância fundamental na mediação das interações ou troca de mensagens. A Equação 2 apresenta a centralidade de intermediação.

$$
B(e)=\sum_{u \in V, v \in V} \frac{\sigma_{e}(u, v)}{\sigma(u, v)}
$$

Equação 2. Equação para Centralidade de Intermediação

\subsubsection{Centralidade de Proximidade (Closeneess Centrality)}

Através desta métrica, Closeneess Centrality, é possível certificar o quão rápido um nó pode interagir com os demais, pois quanto menor a distância total referente à soma dos menores caminhos de um com o demais, maior é sua aproximação perante todos [Oliveira 2013], e consequentemente, mais central é o nó. A Centralidade de Proximidade pode ser definida através da Equação 3 seguinte.

$$
C_{C}\left(n_{i}\right)=\left[\sum_{j=1}^{g} d\left(n_{i}, n_{j}\right)\right]^{-1}
$$

Equação 3. Equação para Centralidade de Proximidade

\section{Correlação Linear}

Frequentemente, em pesquisas, busca-se comprovar a existência de relação entre duas ou mais variáveis, ou seja, saber se a alteração no valor de uma variável provoca alterações no valor de outra variável. Para Levin (2012) as correlações variam com relação à sua força e podem ser visualizadas através de um gráfico de dispersão, que mostra o modo como os escores, em qualquer uma das variáveis, X e Y, estão dispersos através de uma gama de valores de escores possíveis. Assim, a intensidade da correlação entre as variáveis aumenta à medida que os pontos em um gráfico de dispersão formam algo mais parecido com uma reta diagonal através do centro da dispersão de pontos no gráfico.

Quanto à direção, a correlação pode ser descrita como positiva ou negativa, e ambas representam um tipo de relação linear. Uma correlação positiva indica que uma vez percebidos altos escores na variável $\mathrm{X}$, a variável $\mathrm{Y}$ também tende a receber altos escores [Levin, 2012]. Por outro lado, uma vez que percebe-se baixos escores em uma variável $\mathrm{X}$, a variável Y também tende a ter baixos escores. Uma correlação negativa existe quando obtêm-se altos escores na variável $\mathrm{X}$ e, consequentemente, baixo escores na variável $\mathrm{Y}$, ou, em contrapartida, é percebido baixos escores em X, informando assim que $\mathrm{Y}$ tende a alcançar altos escores.

Já o coeficiente de correlação linear mede e expressa numericamente a intensidade e a direção da relação linear entre duas variáveis quantitativas. Chamado também de Coeficiente de Correlação de Pearson (r), proposto por Karl Pearson [Clark 2011]. Tal coeficiente é encontrado através da Equação 4 a seguir. 


$$
\frac{n \sum x y-\left(\sum x\right)\left(\sum y\right)}{\sqrt{\left[n \sum x^{2}-\left(\sum x^{2}\right)\right] \cdot\left[n \sum y^{2}-\left(\sum y^{2}\right)\right]}}
$$

Equação 4. Equação do Coeficiente de Correlação de Pearson

\section{Descrição do Experimento}

O experimento foi conduzido no AVA Moodle em um período de dezesseis semanas e seis módulos, com uma amostra de 513 participantes (sendo 508 alunos, 4 tutores e 1 professor) da disciplina Introdução à Computação de um curso técnico de uma Instituição de Ensino Federal. Dentre os 508 alunos matriculados no curso foi observado, ainda, um índice de evasão equivalente a 39\% (196 alunos). Desta forma são considerados na análise deste estudo apenas 312 alunos participantes, equivalente a $61 \%$ do total de alunos matriculados no curso.

O curso analisado foi organizado em seis módulos. O primeiro, segundo, terceiro e quarto módulos compreenderam atividades síncronas e assíncronas ao longo de duas semanas, para cada módulo. No quinto e sexto módulos as atividades foram dispostas em quatro semanas, para cada módulo. É importante ressaltar que dentre as atividades oferecidas aos alunos no decorrer do curso, a ferramenta de comunicação assíncrona fórum de discussão esteve disponível em todas as semanas dos módulos, propiciando a constante interação entre os alunos e entre os alunos com os tutores ou professor.

Para estabelecer a correlação existente entre o desempenho do aluno com a sua conduta nos fóruns de discussão foi utilizado o método estatístico de correlação para identificar a intensidade da relação entre estas variáveis. A técnica de correlação foi escolhida devido às características do experimento em questão: tamanho da amostra, nível de exigência esperado do teste, necessidade de avaliar a relação entre duas medidas quantitativas e de descrever a relação entre as variáveis.

Todas as interações são persistidas no Moodle e os relatórios de participação nos fóruns de discussão foram extraídos de forma manual devido a inexistência de uma ferramenta de extração de dados compatível com a versão do AVA Moodle adotado para a pesquisa.

\section{Resultados alcançados}

Após a extração dos dados foi utilizado para a obtenção do grafo a ferramenta Gephi, para visualização e aplicação das métricas de ARS. A rede completa dos dados analisados com as interações nos fóruns de discussão de todo o período do curso pode ser visualizada em https://sites.google.com/site/grafosdosforunsdediscussao/. Ressalvamos que os nós referem-se aos alunos, tutores e/ou professores que participam do fórum e as arestas correspondem as interações (ligações) estabelecidas entre eles. Ao juntar os dados extraídos dos fóruns de discussão e agrupá-los em uma rede, a ferramenta identificou a existência de nove comunidades ou sub-redes, indicando cada um dos polos/turmas do curso. 
Cada cor, no grafo, tem uma correspondência em relação aos polos/turmas do curso: Cinza - turma T1; Vermelho - turma T2; Amarelo - turma T3; Verde - turma T4; Roxo - turma T5; Rosa - turma T6; Laranja - turma T7; Azul - turma T8; Marrom turma T9. Percebe-se ainda que no centro do grafo existe uma junção entre os nós nas diversas cores. Nesta porção encontram-se os nós que interagiram com mais de uma turma, que são os tutores que interagem com alunos de diversas turmas e o professor-formador da turma como nó central.

A partir da análise realizada com os dados obtidos em cada módulo do curso foi possível perceber características individuais de relacionamento e desempenho identificadas em cada aluno no decorrer das semanas. Os resultados observados ao longo do curso foram comparados para podermos acompanhar com mais nitidez o desenvolvimento das interações nos fóruns de discussão e a correlação entre os índices de desempenho dos alunos selecionados através da amostra e as métricas de ARS supracitadas. A evolução dos grafos gerados em cada módulo pode ser visualizado em https://sites.google.com/site/grafosdosforunsdediscussao/.

O primeiro, o segundo, o terceiro e o quarto módulo apresentam as interações encontradas nos fóruns de discussão referentes a duas semanas do curso, cada. Já o quinto e o sexto módulo são compostos de quatro semanas, cada. É possível notar na Figura 2 uma disparidade de interações em cada módulo. Nos módulos iniciais, por exemplo, é percebido uma maior quantidade de participação. Enquanto que nos módulos finais essa frequência diminui consideravelmente. Tal informação nos leva a identificar, ligeiramente, em que momento os alunos começaram a deixar de interagir nas atividades do curso.

Em face disto podemos acompanhar a evolução dos índices de correlação entre o desempenho e o "Degree" do aluno no módulo. A Figura 3 informa que em todos os casos a correlação é positiva, representando assim um tipo de relação linear. Descritos graficamente, os pontos nos gráficos de dispersão tendem sempre a formar uma linha reta imaginária que cruza os pontos no gráfico e que se agrupam em torno da linha imaginária em direção crescente. Portanto, esta correlação indica que quanto maior o "Degree", número de interações estabelecidas por um aluno (representado por um nó na rede) no grafo, maiores são as chances de o aluno alcançar um bom desempenho no módulo. 
V Congresso Brasileiro de Informática na Educação (CBIE 2016)

Anais dos Workshops do V Congresso Brasileiro de Informática na Educação (CBIE 2016)
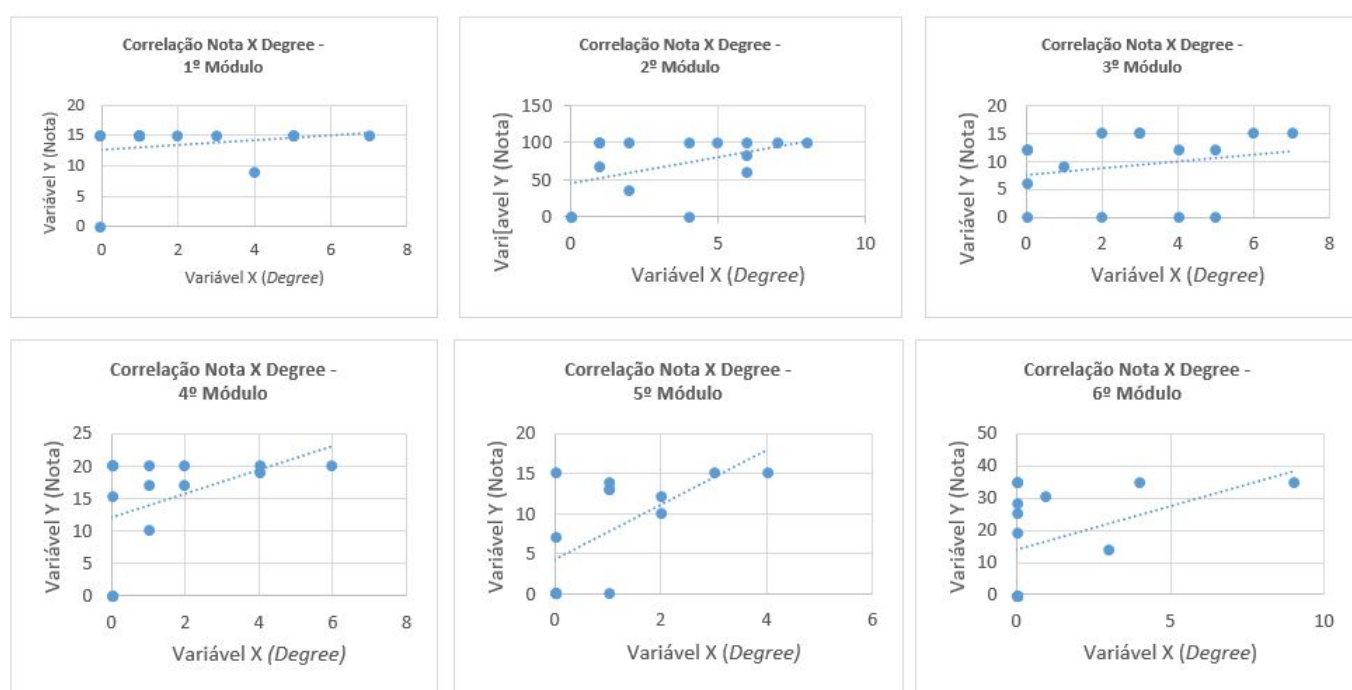

Figura 3. Evolução dos índices de correlação entre o desempenho (nota) e o Degree

Na Figura 4 acompanhamos a evolução dos índices de correlação entre o desempenho e o "Closenneess” do aluno no módulo. Descritos graficamente, os pontos nos gráficos de dispersão informam uma correlação positiva entre as variáveis e expressam coeficientes de correlação positiva, ora fraca, ora moderada, nos módulos. Assim, esta correlação indica que os nós que aparecem no caminho mais curto entre os demais nós da rede tem maiores chances de alcançar um bom desempenho nas atividades avaliativas propostas.

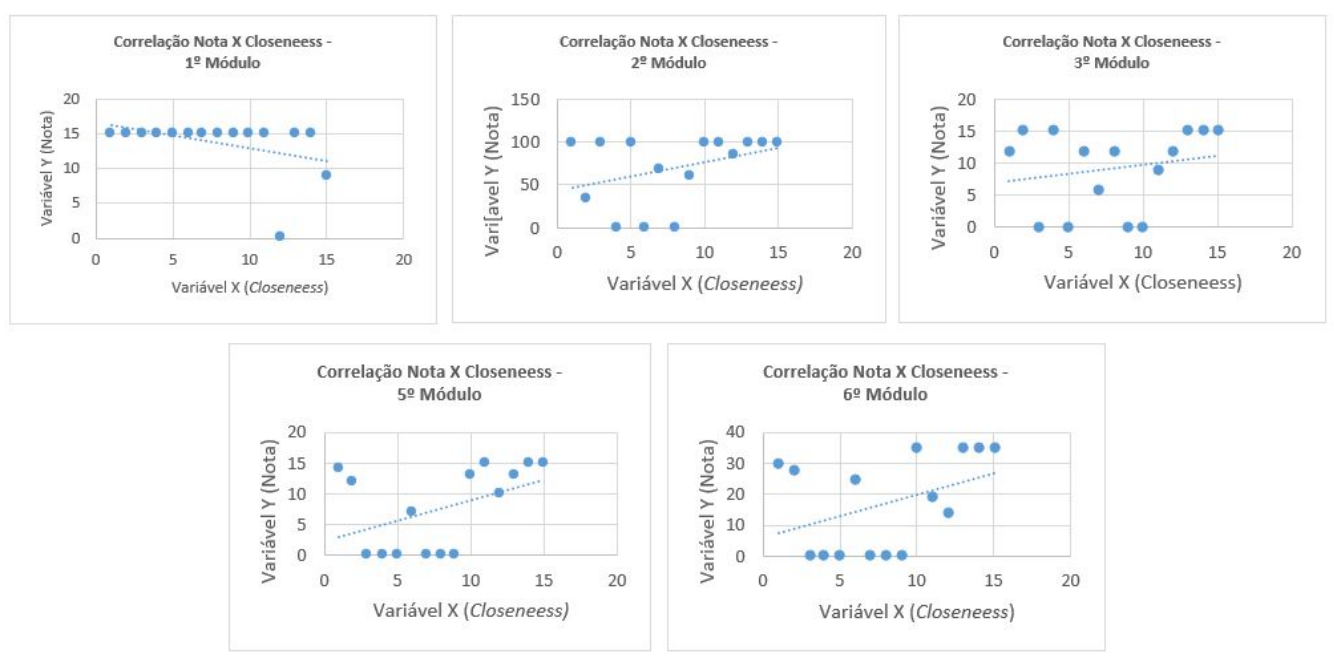

Figura 4. Evolução dos índices de correlação entre o desempenho (nota) e o Closenneess

Os gráficos de dispersão para a métrica Betweenness, bem como os coeficientes de correlação linear são omitidos neste trabalho, pois sua correlação com a nota nos módulo do curso foi tão baixa que todos seus valores tenderam a zeram, impossibilitando a análise precisa destes dados.

\subsection{Análise geral dos dados obtidos}


V Congresso Brasileiro de Informática na Educação (CBIE 2016)

Anais dos Workshops do V Congresso Brasileiro de Informática na Educação (CBIE 2016)

Percebemos que durante toda a disciplina a turma que mais participou dos fóruns de discussão foi a turma T2 com $15.06 \%$ de participação. Logo após, a turma T3 com $12.82 \%$ de interação no fórum, seguidos da turma T4 com 12.18\% e das turmas T6 e T9 com $11.86 \%$ de participação, cada. As demais turmas apresentaram índices inferiores a estes (T7 - 9.94\%; T5 - 9.62\%; T1 - 8.65\%; T8 - 8.01\%).

Para esta análise são considerados 15 alunos de diferentes polos de acordo com as variações de desempenho percebidas e com os índices de participação registrados nos relatórios dos fóruns de discussão, selecionados pela amostra não aleatória que utiliza a amostragem intencional [Levin 2012]. Assim, podemos analisar na Tabela 1 as métricas de Centralidade de Grau, de Intermediação e de Proximidade (Closenness) calculadas para os quinze alunos elencados não aleatoriamente na amostragem.

Tabela 1. Análise a partir da métricas de ARS para a rede completa do curso

\begin{tabular}{|c|c|c|c|c|c|}
\hline ID do aluno & ID da turma & Degree & Closeneess & Betweenness & Média no curso \\
\hline A1 & $\mathrm{T} 2$ & 14 & 0.286 & 0.001 & 79 \\
\hline A2 & T3 & 16 & 0.368 & 0.087 & 82.6 \\
\hline A3 & $\mathrm{T} 2$ & 13 & 0.283 & 0.005 & 92 \\
\hline A4 & T6 & 14 & 0.204 & 0.003 & 92 \\
\hline A5 & T9 & 13 & 0.381 & 0.012 & 84.4 \\
\hline A6 & $\mathrm{T} 4$ & 5 & 0.281 & 0 & 93 \\
\hline A7 & $\mathrm{T} 7$ & 5 & 0.293 & 0 & 42.91 \\
\hline A8 & T1 & 12 & 0.203 & 0.002 & 27 \\
\hline A9 & T9 & 8 & 0.314 & 0 & 4.5 \\
\hline A10 & T5 & 1 & 1 & 0 & 88.2 \\
\hline A11 & $\mathrm{T} 2$ & 1 & 1 & 0 & 6.3 \\
\hline A12 & T4 & 1 & 0.22 & 0 & 37.2 \\
\hline A13 & T1 & 1 & 0.169 & 0 & 4.5 \\
\hline A14 & T9 & 9 & 0.293 & 0.031 & 52.69 \\
\hline A15 & T6 & 2 & 0.203 & 0 & 47.76 \\
\hline
\end{tabular}

De um ponto de vista mais geral, observamos que ao final da disciplina os alunos que apresentaram um melhor desempenho (A1, A2, A3, A4 e A5) foram aqueles que realizaram um maior número de interações na rede, ou seja, que apresentaram um maior Degree. Por outro lado, ainda quanto ao Degree, percebemos também que os alunos A6 e A10 não interagiram com frequência nos fóruns de discussão, mas obtiveram um bom desempenho ao final da disciplina. Um outro caso interessante a referir é o do aluno A14 que participou dos fóruns um número considerável de vezes, realizou a avaliação final na disciplina e obteve aprovação na mesma. Enquanto que o aluno A8 mesmo tendo interagido muitas vezes não foi aprovado na disciplina.

Quanto ao Closeneess notamos que os menores índices foram dos alunos A13, A15 e A8. Ambos não alcançaram um bom desempenho na disciplina, chegando à 
V Congresso Brasileiro de Informática na Educação (CBIE 2016)

Anais dos Workshops do V Congresso Brasileiro de Informática na Educação (CBIE 2016)

reprovação. Mas, um caso interessante a destacar foi o aluno A4 que também apresentou um índice de proximidade baixo e que obteve um bom desempenho na disciplina. Essas características denotam que os referidos alunos são os mais próximos dos demais nós da rede e que sua proximidade influenciou negativamente em seu desempenho, em alguns casos. Por outro lado, percebemos que aqueles (A2 e A5, por exemplo) que obtiveram um maior índice em Closeneess alcançaram um bom desempenho.

Os maiores índices em Betweenneess foram dos alunos A2, A5 e A14 que também apresentaram um bom desempenho no curso. Sendo que este último aluno precisou realizar uma avaliação final para alcançar aprovação na disciplina. Através dessa métrica foi possível perceber os alunos que agem como ponte ao longo do caminho entre dois outros nós, ou seja, aqueles que contribuem e intermediam a propagação da informação. Coincidentemente, os mesmos alunos que possuíram um número maior de Degree também foram aqueles que apresentaram um maior índice em Betweenneess.

Então, correlacionamos o desempenho do aluno (variável Y), expresso pela média na disciplina, e o índice em Degree. Com esta análise foi expressa a existência de uma correlação positiva quanto a direção do gráfico de dispersão. Entretanto, quando demonstrado numericamente a intensidade e a direção da correlação linear entre as variáveis supracitadas obtivemos um coeficiente de correlação equivalente a -0,1489101, indicando que as variáveis estão moderadamente correlacionadas. Não foi possível obter o gráfico de dispersão para as métricas Betweenness e Closenness, bem como seus respectivos coeficientes de correlação linear. Assim essas informações são omitidas neste trabalho, pois os coeficientes de correlação com a média dos alunos foi tão baixa que todos seus valores tenderam a zeram.

\section{Considerações Finais e Trabalhos Futuros}

Com base nos dados apurados, é possível afirmar que este estudo mostrou-se satisfatório, uma vez que além de demonstrar a possibilidade de uso de métricas de ARS aplicadas ao contexto da EAD, conseguiu demonstrar, também, a correlação existente entre os índices de participação obtidos por meio das métricas de ARS e o desempenho dos alunos, mesmo analisando apenas a troca de mensagens nos fóruns, sem considerar outras possíveis variáveis que podem ter influência sobre o resultado do processo.

Estimativas sobre a correlação entre as interações nos fóruns e o desempenho dos alunos, observados ao longo da disciplina, podem ser úteis para professores acompanharem individualmente estudantes e desenvolverem meios pedagógicos que busquem minimizar reprovações e, até, possíveis evasões no curso. Essas informações disponibilizadas em módulos iniciais do curso podem ser utilizadas para 0 desenvolvimento de ações que envolvam alunos de turmas em andamento e não somente para futuras turmas. Os padrões descobertos podem ser úteis, ainda, como informação para apoiar os professores no desenvolvimento de estratégias para monitoramento e gerenciamento de cursos a distância.

Para tanto, são propostas futuras a realização do monitoramento das interações em fóruns de discussão através da aplicação de métricas que medem o grau de coesão e o grau de heterogeneidade [Medeiros 2013] - como indicadores da colaboração no grupo; 
V Congresso Brasileiro de Informática na Educação (CBIE 2016)

Anais dos Workshops do V Congresso Brasileiro de Informática na Educação (CBIE 2016)

a análise da interação em fóruns de discussão de diferentes disciplinas/turmas de um mesmo semestre letivo a fim de comparar os resultados obtidos quanto a participação e desempenho das turmas; e, o desenvolvimento de um software ou plugin que viabilize a extração dos dados de fóruns de discussão no AVA Moodle.

\section{Referências Bibliográficas}

Clark, J.; Downing, D. (2011) Estatística Aplicada. 3 ed. São Paulo: Saraiva.

Carvalho, J. S. (2009) Redes e comunidades virtuais de aprendizagem: elementos para uma distinção. página 196. Dissertação de Mestrado. Universidade de São Paulo, São Paulo.

Engel, A.; Coll, C.; Bustos, A. (2013) Distributed Teaching Presence and communicative patterns in asynchrouns learning: Name versus reply networks. International Journal Computer and Science.

Gottardo, E.; Noronha, R. V. (2012) Social networks applied to distance education courses: analysis of interaction in discussion forums. WebMedia '12: Proceedings of the 18th Brazilian symposium on Multimedia and the web. páginas 355-358.

Levin, J.; Fox, A. J.; Forde, D. R. (2012) Estatística para Ciências Humanas. 11 ed. São Paulo: Pearson Education do Brasil.

Marteleto, M. (2001) Análise de Redes Sociais - aplicações nos estudos de transferência da informação. Ci. Inf. Brasília, Brasil, PP 1-10.

Medeiros, F.; Gomes, A.; Amorim, R.; Medeiros, G. (2013) Redesigning Collaboration Tools to Enhance Social Presence in Online Learning Environments. In Collaboration and Technology. Springer Berlin Heidelberg. páginas 175-191.

Oliveira, R.; Araújo, J.; Medeiros, F.; Brito, A. (2012) Monitoramento das Interações dos Aprendizes na Rede Social Twitter como Apoio ao Processo de Mediação Docente. Brazilian Workshop on Social Network Analysis and Mining, BrasNAM. XXXII Congresso da Sociedade Brasileira de Computação, CSBC. Curitiba, PR.

Oliveira, R. F. (2013) Análise quantitativa das relações entre usuários no Twitter. Dissertação de Mestrado. Universidade Federal da Paraíba, João Pessoa-PB.

Rabbany, R.; TAkaffoli, M.; Zaane, O. R. (2012) Social network analysis and mining to support the assessment of on-line student participation. SIGKDD Explorations Newsletter, Volume 13 Issue 2. páginas 20-29.

Silva, A.; Figueira, A. (2012) Depicting online interactions in learning communities. Global Engineering Education Conference (EDUCON), IEEE.

Silva, J. C.; Brito, A. V.; Medeiros, F. P. A. (2015) Mapeamento Sistemático da Literatura acadêmico-científica sobre Análise de Redes Sociais aplicada em E-Learning. Revista Brasileira de Informática na Educação, RBIE. v. 23, n. 1. DOI 10.5753. 